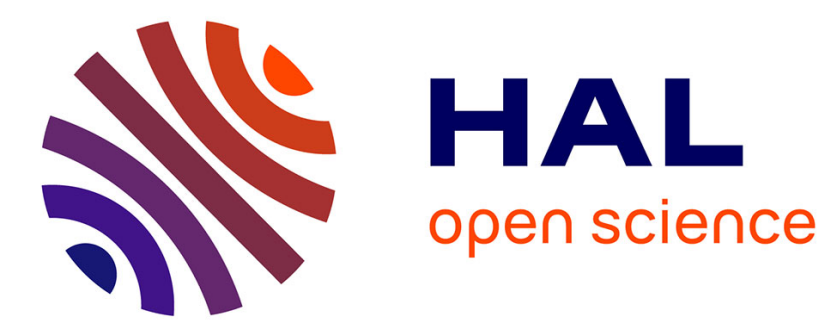

\title{
Croissance du trèfle violet (Trifolium pratense L.) en association avec du ray-grass d'Italie (Lolium multiflorum Lam. ssp. italicum). I. - Description de l'organisation morphologique du trèfle (1)
}

\author{
Jean-Paul Maitre, Louis Assemat, Pierre Jacquard
}

\section{To cite this version:}

Jean-Paul Maitre, Louis Assemat, Pierre Jacquard. Croissance du trèfle violet (Trifolium pratense L.) en association avec du ray-grass d'Italie (Lolium multiflorum Lam. ssp. italicum). I. - Description de l'organisation morphologique du trèfle (1). Agronomie, 1985, 5 (3), pp.251-260. hal-00884756

\section{HAL Id: hal-00884756 https://hal.science/hal-00884756}

Submitted on 1 Jan 1985

HAL is a multi-disciplinary open access archive for the deposit and dissemination of scientific research documents, whether they are published or not. The documents may come from teaching and research institutions in France or abroad, or from public or private research centers.
L'archive ouverte pluridisciplinaire HAL, est destinée au dépôt et à la diffusion de documents scientifiques de niveau recherche, publiés ou non, émanant des établissements d'enseignement et de recherche français ou étrangers, des laboratoires publics ou privés. 


\title{
Croissance du trèfle violet (Trifolium pratense L.) en association avec du ray-grass d'Italie (Lolium multiflorum Lam. ssp. italicum). I. - Description de l'organisation morphologique du trèfle $\left({ }^{1}\right)$
}

\author{
Jean-Paul MAITRE, Louis ASSEMAT* \& Pierre JACQUARD* ${ }^{2}$ ) \\ Ecole Nationale d'Ingénieurs des Travaux Agricoles, Chaire d'Agronomie, 21, bd O.-de-Serres, B.P. 48, \\ F 21800 Quetigny \\ * C.N.R.S., Unité de Biologie des Populations et des Peuplements, Centre Louis Emberger, B.P. 505I, \\ route de Mende, $F 34033$ Montpellier Cedex
}

Des recherches ont été conduites sur la variation des populations d'organes chez le trèfle violet, cultivé pur ou en différentes conditions de concurrence. Les dispositifs sont installés soit en serre, soit en champ; dans ce dernier cas, densité et composition sont parmi les variables contrôlées permettant d'apprécier la dynamique des mélanges. Les observations sont effectuées sur des individus (et modules par la suite), un pied de trèfle violet pouvant être considéré comme une colonie de modules dont chacun a potentiellement un cycle biologique complet. Ces observations ont permis de suivre la variation dans le temps de l'organisation morphologique : ensemble de mođules ayant atteint des étapes différentes de leur «itinéraire biologique ». On a comparé l'état végétatif et l'état reproducteur et apprécié l'effet d'une coupe sur l'organisation ultérieure. La structure de la touffe de trèfle violet est alors le résultat de l'interaction entre la proportion respective de chaque type de module présent avant la coupe et la hauteur de la coupe.

Mots clés additionnels : Morphogenèse, module, état végétatif, état reproducteur, coupe.

Growth of red clover (Trifolium pratense L.) in association with Italian rye-grass (Lolium multiflorum Lam. spp. italicum). I. - Morphological organization of the clover plant.

Variation in the population of organs of the red clover plant was studied in pure stands and in clover-grass mixtures. Trials were set up in the glasshouse and in the field ; in the latter, density and proportion were among the controlled variables used to evaluate the dynamics of the mixtures. Observations were made on individuals (and subsequently on modules) ; a shoot of red clover was treated as a colony of modules, of which any module has the potentiality for a whole biological cycle. These observations allowed us to trace the morphological organization of the plant, i.e. the change with time of a set of modules at different stages of their « biological trajectory ». Vegetative and reproductive stages were compared and the effect of cutting on subsequent morphological organization was evaluated. The structure of a shoot of red clover is then the result of interaction between the respective proportion of each category of module present before cutting and the cutting height.

Additional key words : Morphogenesis, module, vegetative stage, reproductive stage, cutting.

(1) Recherche financée en partie par la DGRST, décisions d'aide n V.I.13.77.1861 et 1862, dans le cadre de l'action incitative « Liaisons Recherche-Enseignement Supérieur dans les disciplines agronomique et vétérinaire ».

${ }^{\left({ }^{2}\right)}$ Avec la collaboration de J.-L. GAUjard et Y. BAILLY, étudiants de l'E.N.I.T.A. 


\section{INTRODUCTION}

La biomasse agronomiquement récoltable d'une population végétale cultivée n'est que la résultante d'une série de transformations structurelles concernant chaque individu de la population. Les événements qui se succèdent dans une culture ne sont pas les mêmes à un moment donné d'un individu à l'autre, même si tous les individus sont génétiquement identiques et débutent leur morphogenèse au même instant. Cette constatation oblige l'agronome à aborder l'étude des modifications quantitatives et qualitatives à l'échelle des populations d'individus et à l'échelle des populations d'organes. Les termes de croissance et de développement habituellement utilisés pour une plante n'ont alors plus la même acception.

L'approche classique des études de croissance utilise les accroissements de poids et de surface pour la mise au point de modèles fondés sur « le taux de croissance relative " ou "le taux d'assimilation nette ». Depuis les premiers travaux de GREGORY (1917) et de BLACKMAN (1919), de nombreux auteurs ont développé et perfectionné l'analyse de la croissance végétale. Des études synthétiques ont été fournies à ce sujet par RADFORD (1967), EVANS (1972) et HUNT (1978a).

L'analyse démographique de la croissance est plus récente. Pour BAZZAZ \& HARPER (1977), la croissance d'une plante peut être décrite de façon précise par des paramètres démographiques. En effet, HARPER (1977) considère que la plante est un ensemble d'unités de structures greffées les unes sur les autres, indépendantes et réitérées (la feuille et son bourgeon axillaire, par exemple) ; il assimile donc la croissance de la plante entière à celle d'une population d'organes. L'exemple le plus typique est celui des graminées dont la touffe ne fonctionne pas comme une individualité mais comme une colonie de talles soumises à diverses interactions (GILlET, 1970 ; GILlET, 1980). BAZZAZ \& HARPER (1977) montrent (chez Linum usitatissimum L.) que des paramètres démographiques (espérance de vie, classe d'âge, natalité, mortalité...) peuvent être calculés à partir de résultats sur la naissance et la mort des feuilles. Ceci permet une interprétation fine de la réponse de la plante aux facteurs de l'environnement (MAITRE, 1981).

L'approche démographique ne peut être opposée aux conceptions traditionnelles d'analyse de croissance que dans sa nature mais pas dans son efficacité.

Pour HUNT (1978b), les 2 approches sont complémentaires et peuvent être utilisées selon ce que l'expérimentateur recherche.

Une $3^{\mathrm{e}}$ approche, due à MALLET (1979), utilise l'étude de diverses caractéristiques statistiques (variance, coefficient de dissymétrie) des distributions de mesures de croissance pour tester le niveau de croissance et ses éventuelles perturbations par rapport à un potentiel génétique. Ce modèle serait applicable en particulier à une population d'organes déterminés.

Nous nous proposons, dans ce $1^{\mathrm{er}}$ article, de tenter une description de la construction de la plante de trèfle violet puis, dans un $2^{\mathrm{e}}$, de la plasticité de son organisation morphologique sous le contrôle du milieu par l'utilisation d'analyses démographiques comme le préconise HARPER (1977). Cette description sera entreprise en tenant compte des conditions culturales et notamment du stade de récolte habituellement fixé entre le bourgeonnement et le début de floraison.

\section{MATÉRIEL ET MÉTHODES}

Les méthodes utilisées reposent sur 2 types d'investigations : des mesures morphogénétiques, des observations démographiques.

La présentation de l'organisation morphologique et de ses variations s'appuie sur les résultats obtenus dans 3 expériences utilisant la concurrence interspécifique (association avec du ray-grass d'Italie) et intraspécifique (culture pure), comme facteur de variation des populations d'organes.

Dans ces expériences, les feuilles de trèfle sont individualisées par des bagues colorées, le nombre de plantes étudiées par traitement expérimental variant selon les expériences entre 20 et 60 .

Le marquage des feuilles permet de noter :

- la date d'émergence de la feuille (fig. 1),

- la date de mort de la feuille,

- l'ordre d'émergence,

- la filiation foliaire.

Le nombre de feuilles ainsi que son évolution dans le temps sont ainsi déterminés.

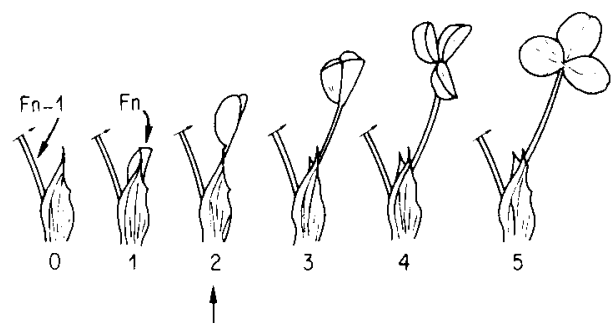

Figure 1

Stades repères adoptés pour l'émergence d'une feuille d'ordre $n$ $\left(F_{n}\right)$, Trifolium pratense $L$.

Critical stages for leaf emergence.

$0:$ Folioles non visibles; leaflets hidden.

I: Folioles en partie cachées par la stipule de la feuille précédente; leaflets partly hidden by the stipule of the preceding leaf.

2 : Folioles dégagées mais non ouvertes, pétiole non visible; leaflets emerged but not open, petiole hidden.

3 : Folioles dégagées et écartées, pétiole visible; leaflets emerged and separated, petiole visible.

4: Folioles à demi-ouvertes; leaflets semi-open.

5 : Folioles ouvertes, limbe étalé ; leaflets open, lamina displayed.

La $I^{\text {re }}$ expérience a porté sur l'étude de 3 familles consanguines de trèfle violet testées lors de travaux antérieurs pour leur aptitude à la vie en association (MAITRE, 1977).

La $2^{2}$ expérience (MAITRE \& JACQUARD, 1980), réalisée au champ, mérite une description plus détaillée. La densité et la composition (séries de remplacement de DE WIT, 1960) ont été les 2 facteurs contrôlés permettant d'apprécier la dynamique des mélanges.

Mis en place au début du mois d'avril 1978, l'essai a été suivi jusqu’à la fin de juillet 1979 , soit pendant 16 mois ( 2 cycles annuels). 


\section{A. Matériel végétal}

Le choix s'est porté sur des cultivars reconnus comme étant productifs : trèfle violet cv. "Rotra » (tétraploïde), ray-grass d'Italie cv. « Adret » (diploïde, non alternatif).

\section{B. Dispositif}

Le plan expérimental comporte 3 répétitions des traitements suivants (soit 15 conditions de culture, 9 associations et 6 cultures pures) :

Série de remplacement

\begin{tabular}{|c|c|c|c|c|c|c|}
\hline $\begin{array}{l}\text { Densité } \\
\text { de semis }\end{array}$ & $\begin{array}{l}\text { T. viol. } \\
\text { R. ital. }\end{array}$ & $\begin{array}{r}0 \\
100\end{array}$ & $\begin{array}{l}25 \\
75\end{array}$ & $\begin{array}{l}50 \\
50\end{array}$ & $\begin{array}{l}75 \\
25\end{array}$ & $\begin{array}{r}100 \\
0\end{array}$ \\
\hline$\left(\right.$ plantes $\left./ \mathrm{m}^{2}\right)$ & & $\mathrm{T}_{0}$ & $T_{1}$ & $\mathbf{T}_{2}$ & $T_{3}$ & $\mathrm{~T}_{4}$ \\
\hline $\begin{array}{l}100\left(\mathrm{D}_{1}\right) \\
400\left(\mathrm{D}_{2}\right) \\
700\left(\mathrm{D}_{3}\right)\end{array}$ & & $\begin{array}{l}\mathrm{T}_{0} \mathrm{D}_{1} \\
\mathrm{~T}_{0} \mathrm{D}_{2} \\
\mathrm{~T}_{0} \mathrm{D}_{3}\end{array}$ & $\begin{array}{l}\mathrm{T}_{1} \mathrm{D}_{1} \\
\mathrm{~T}_{1} \mathrm{D}_{2} \\
\mathrm{~T}_{1} \mathrm{D}_{3}\end{array}$ & $\begin{array}{l}\mathrm{T}_{2} \mathrm{D}_{1} \\
\mathrm{~T}_{2} \mathrm{D}_{2} \\
\mathrm{~T}_{2} \mathrm{D}_{3}\end{array}$ & $\begin{array}{l}\mathrm{T}_{3} \mathrm{D}_{1} \\
\mathrm{~T}_{3} \mathrm{D}_{2} \\
\mathrm{~T}_{3} \mathrm{D}_{3}\end{array}$ & $\begin{array}{l}T_{4} D_{1} \\
T_{4} D_{2} \\
T_{4} D_{3}\end{array}$ \\
\hline
\end{tabular}

La densité $\mathrm{D}_{2}$ est couramment pratiquée.

L'agencement des partenaires, dans l'association, est réalisé ligne à ligne, l'unité parcellaire étant constituée de 12 lignes dont 8 lignes récoltables (fig. 2).

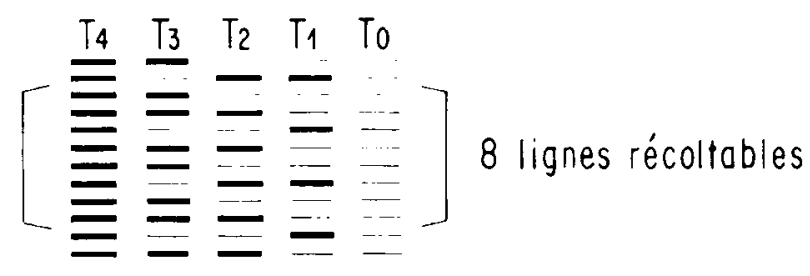

Figure 2

$2^{e}$ expérience: unité parcellaire constituée de 12 lignes dont 8 récoltables.

$2^{\text {nd }}$ experiment : plot unit of 12 rows ( 8 harvested rows).

\section{Observations et mesures}

\section{En végétation}

Après tirage au sort, 10 individus par parcelle sont repérés sur les 2 lignes centrales de trèfle au moyen d'étiquettes et de bagues. Chaque individu fait l'objet de notations tous les 2 jours.

\section{A la récolte}

Parmi les 10 individus de trèfle violet, ceux numérotés de 1 à 5 sont récoltés en mélange, avec séparation des limbes, pétioles et tiges. Les individus 6 à 10 sont récoltés de même, mais dans ce cas, les limbes sont séparés par classes d'âge. On obtient ainsi, notamment, la biomasse (M.S.) de chaque ordre de feuille. Les pétioles et les tiges, tous individus confondus, sont mis en étuve pour l'obtention de la matière sèche. Des photocopies des limbes sont passées à l'intégrateur de surfaces. Le complément des individus non bagués pour le trèfle et la totalité du ray-grass sont récoltés par parcelle. La matière fraîche est passée en étuve à $105^{\circ} \mathrm{C}$ pour l'obtention du poids sec.

\section{Relevés climatiques}

Ils ont concerné : les températures minimales et maximales, l'humidité relative, l'évaporation (Piche), l'ensoleillement, les précipitations.

L'analyse a porté sur les conditions de culture, combinant la densité et la proportion de ray-grass d'Italie associé. Ces résultats concernant la biomasse portent sur 5 coupes $\left(C_{1}, C_{2} \ldots C_{5}\right)$ alors que les aspects démographiques et morphogénétiques n'ont pu être étudiés que pour 4 cohortes, c'est-à-dire pour les organes nés entre le semis et la $1^{\text {re }}$ coupe, ou entre les coupes $C_{2}$ et $\mathrm{C}_{3}, \mathrm{C}_{3}$ et $\mathrm{C}_{4}, \mathrm{C}_{4}$ et $\mathrm{C}_{5}$, constituant alors les repousses. Les données relatives à la $2^{\mathrm{e}}$ cohorte $\left(C_{1}-C_{2}\right)$ n'ont pu être analysées, le nombre de plantes étant insuffisant. Lorsque cela a été possible les équations des courbes de réponse ont été calculées. Les 2 premières séries de résultats ont été utilisées pour la détermination des relations entre organisation et climat.

La $3^{\mathrm{e}}$ expérience (non publiée), concernait l'étude de la croissance au champ du cv. "Rotra » cultivé pur et en association avec le ray-grass d'Italie.

\section{RÉSULTATS}

\section{A. La conception "modulaire" et ses implications} (tabl. 1)

Il nous a paru nécessaire de nous étendre, ici, sur le langage particulier des démographes, appliqué aux végétaux, la notion de module pouvant, en effet, être assez fructueuse pour les agronomes.

\section{TABLEAU 1}

Définition des termes particuliers utilisés dans ce travail. Glossary of specific terms used in the work.

Cohorte (d'organes) : Ensemble d'organes vivant un niême événement démographique pendant une même durée de temps. Ex. : dans cet article, ensemble des organes nés entre 2 coupes.

Emergence (d'une feuille) : Moment où les folioles du limbe sont entièrement dégagées de la stipule précédente, le pétiole n'étant pas encore visible.

Filiation de modules (ou de feuilles) : Liaison de modules (ou de feuilles) résultant les uns des autres.

Lignée de modules: Ensemble de modules résultant les uns des autres.

Module: Unité de structure indépendante et réitérée, dont l'ensemble, la plante entière, constitue une population ou colonie.

Ordre d'un module (ou d'une feuille) : Rang lié à l'émergence sur la plante.

Précocité d'émergence (foliaire) : Dans des conditions environnementales données, la précocité d'émergence se définit comme le temps écoulé depuis le semis, le repiquage ou la dernière récolte jusqu'au moment où la nouvelle feuille émerge de la stipule de la feuille précédente.

Précocité de ramification: Temps écoulé depuis le semis, le repiquage ou la dernière récolte jusqu'au moment où la $1^{\text {re }}$ ramification apparaît.

Secondaire, Tertiaire: $2^{\mathrm{c}}, 3^{\mathrm{c}}$ subdivision d'une lignée d'organes après la division primaire. 


\section{Le concept de module de structure}

Chez $T$. pratense, l'ensemble constitué d'une feuille et du bourgeon axillaire associé peut régénérer la plante entière après séparation de la plante mère puis repiquage. Ce clonage pratiqué en sélection a un intérêt limité par des inconvénients d'ordre pathologique (viroses). Avec HARPER (1977), nous adopterons le terme de « module de structure » pour désigner l'unité d'organisation constituée par la feuille et son bourgeon axillaire. Un pied de trèfle violet peut être considéré comme une colonie de modules dont chacun a potentiellement un cycle biologique complet : naissance ; phase juvénile, de croissance et d'assimilation prépondérante ; multiplication végétative, c'est-à-dire contribution des ressources à la naissance d'autres feuilles donc d'autres modules (réitération); floraison puis production de graines ; sénescence, migration des assimilats prépondérante; mort.

A un instant donné, sous la pression de l'environnement physique et biologique, des conditions culturales et des corrélations entre modules, la touffe de trèfle violet se présente comme un ensemble de modules ayant atteint des étapes différentes de leur « itinéraire biologique $»$.

\section{Les différents types de modules}

Selon la filiation consécutive à la réitération, nous distinguons dans une lignée modulaire (fig. 3), de $1^{\text {re }}$ génération : des modules primaires jouant le rôle de modules principaux (Mi); des modules secondaires issus des modules primaires (mij) ; des modules tertiaires issus de modules secondaires (mijk).

L'émergence de modules quaternaires ne concerne que la plante isolée cultivée sans contrainte ni concurrence.

Un module primaire d'ordre $i$ (Mi) est composé d'une feuille principale ou feuille primaire (Fi) et d'un bourgeon axillaire (Bi) qui pourra donner par la suite un rameau ( $R i)$ possédant des feuilles secondaires (fij) et une tige (Ti) terminée par une inflorescence (Ii). Les feuilles tertiaires (fijk) apparaissent fréquemment en conditions favorables.

Selon l'état d'organisation du module, nous pouvons distinguer 3 degrés de complexité en relation avec l'âge (fig. 3) :

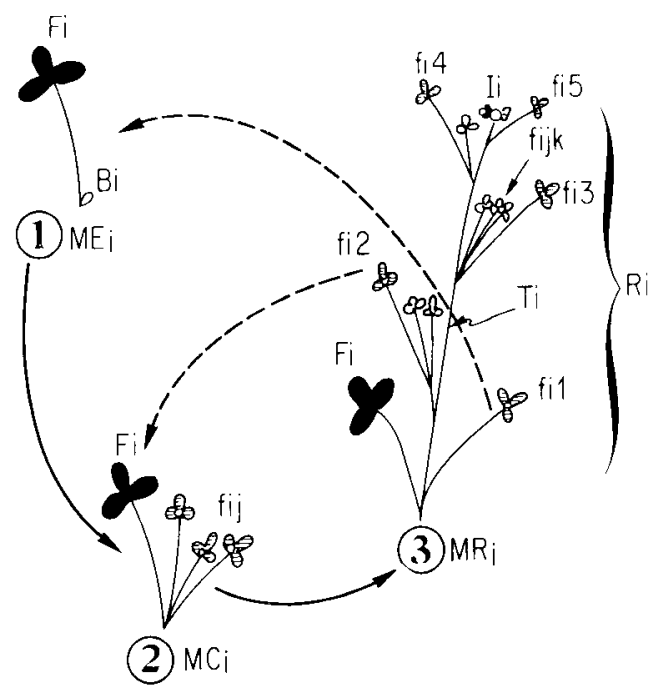

- une phase végétative; nous appelons module végétatif tout module du type :

$$
\begin{aligned}
& \mathrm{MEi}=\mathrm{Fi}+(\mathrm{Bi}) \\
& \text { ou } \\
& \mathrm{MCi}=\mathrm{Fi}+\left(\mathrm{fi}_{1}+\mathrm{bi}_{1}\right)+\left(\mathrm{fi}_{2}+\mathrm{bi}_{2}\right)+\ldots(f \mathrm{fij}+\mathrm{bij})+ \\
& +\ldots+(\mathrm{fin}+\mathrm{bin}) \\
& \text { avec } \\
& (\mathrm{fij}+\mathrm{bij})=\mathrm{mij}
\end{aligned}
$$

La formulation (1) correspond à un module végétatif élémentaire, la formulation (2) correspond à un module végétatif composé. Un module primaire composé (MCi) a donné naissance à autant de modules secondaires (mij) qu'il y a de feuilles secondaires.

- une phase reproductive; nous appelons module reproducteur ou module adulte tout module de type :

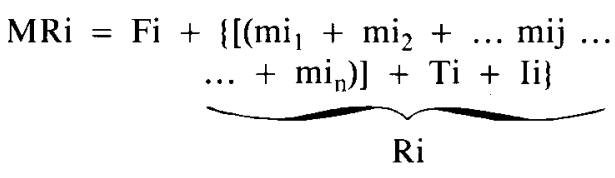

ou encore :

$$
\begin{aligned}
& \mathrm{MRi}=\mathrm{Fi}+\int\left[\left(\mathrm{mi}_{1}+\left(\mathrm{mi}_{11}+\mathrm{mi}_{12}+\ldots\right)+\mathrm{mi}_{2}+\right.\right. \\
& +\left(\mathrm{mi}_{21}+\mathrm{mi}_{22}+\ldots\right) \ldots \\
& \ldots+\underbrace{m i}+\left(m i_{n 1}+\mathrm{mi}_{\mathrm{n} 2}+\ldots\right)]+\mathrm{Ti}+\mathrm{Ii}\} \\
& \text { Ri }
\end{aligned}
$$

Un module primaire reproducteur est obligatoirement composé de modules secondaires et très souvent de modules tertiaires : l'ensemble constituant une lignée modulaire.

Selon cette conception, le passage du module primaire végétatif au module primaire reproducteur se fait par l'acquisition d'un nombre déterminé de modules secondaires en fonction du rang du module primaire. Il faut par exemple 6-7 feuilles secondaires pour que le module primaire $M_{5}$ donne un rameau fleuri $R_{5}$, mais il n'en faut que 5-6 pour le module $\mathbf{M}_{6}$.

Figure 3

Les différents états d'organisation d'un module.

The several stages of organization of a module.

Organes primaires :

$F_{i}:$ Feuille

$B_{i}$ : Bourgeon axillaire

$T_{i}:$ Tige

$I_{i}:$ Inflorescence

$R_{i}:$ Rameau

$M_{E i}:$ Module élémentaire

$M_{C i}:$ Module composé

$M_{R i}$ : Module reproducteur

Primary organs :

Leaf

Axillary bud

Stem

Inflorescence

Branch

Elementary module

Composil module

Reproductive module

Organes secondaires:

fij : feuille

Organes tertiaires :

fijk : feuille

Secondary organs :

leaf

Tertiary organs :

leaf

Formation d'une lignée (3) de 1re génération.

Formation of a Ist generation line.

“Formation d'une lignée de génération ultérieure; dans le cas préventé, la feuille parentale est $f i_{1}$, et $\mathrm{fi}_{2}$.

Formation of a later generation line; the parental leaf is $f i_{1}$ and $f i_{2}$. 
Le trèfle violet, comme beaucoup d'espèces fourragères, présente la faculté de repousser et de refleurir après une coupe. Il s'ensuit que l'architecture de la touffe dépend étroitement des modalités d'exploitation (hauteur, rythme et stade de coupe). Selon le numéro de la coupe on distingue :

- une cohorte de modules de $1^{\text {re }}$ génération correspondant à la période démographique allant du semis à la $1^{\text {re }}$ récolte,

- une cohorte de modules de $2^{\mathrm{e}}$ génération correspondant à la période démographique allant de la $1^{\text {re à }}$ la $2^{\mathrm{e}}$ récolte,

- etc...

La cohorte de $1^{\text {re }}$ génération (ensemble des organes émis du semis à la $1^{\text {re }}$ coupe) se compose de modules primaires (Mi), de modules secondaires (mij), voire de modules tertiaires (mijk). Les cohortes suivantes sont semblables à la première. Elles sont composées d'une suite de modules que l'on peut qualifier de primaires et de secondaires, sans qu'il soit nécessaire de connaître la filiation du module de départ, c'est-à-dire du module de $1^{\text {re }}$ génération qui a échappé à la barre de coupe de la faucheuse ou à la dent de l'animal. Une culture de trèfle violet peut donc être analysée soit comme une population de plantes, soit comme une population de modules, en particulier lorsque l'individualisation des touffes n'est plus possible $\left(2^{\mathrm{e}}\right.$ cohorte et suivantes).

L'application de l'équation fondamentale de la biologie des populations (HARPER, 1977) à la population de modules de trèfle violet sur une surface donnée s'écrit :

$$
\mathrm{M}_{\mathrm{t}+1}=\mathrm{M}_{\mathrm{t}}+\mathrm{n}-\mathrm{m}-\mathrm{E}
$$

$\mathbf{M}_{\mathrm{t}}=$ nombre de modules présents au temps $\mathrm{t}$,

$\mathrm{n}=$ nombre de naissances,

$\mathrm{m}=$ nombre de morts,

$\mathrm{E}=$ nombre de modules exportés. Ces « exportations " correspondent soit aux dégâts occasionnés par des ravageurs animaux (prédation), soit à l'exploitation du fourrage en fauche ou en pâture. Dans ce cas, la perturbation est telle que $\mathrm{E}$ devient très grand et avoisine $M_{t}+n-m$.

\section{B. L'état végétatif}

Contrairement à ce que l'on observe chez d'autres légumineuses comme la luzerne qui émet une tige dès l'acquisition de la $2^{\mathrm{e}}$ feuille trifoliolée (HruSKOVA, 1973), la plantule de trèfle violet s'organise en une rosette d'abord constituée de modules primaires élémentaires, ensuite de modules primaires composés de modules secondaires élémentaires (fig. 4).

L'organisation de la rosette s'effectue en 2 temps : tout d'abord, la plantule n'émet que des feuilles primaires puis, pour un stade compris entre $4 \grave{a} 6$ feuilles primaires, la plante se ramifie en émettant des feuilles secondaires. Cette émission s'effectue de façon centripète : les modules primaires les plus âgés se ramifient en premier, la plupart du temps à partir de $\mathrm{M}_{3}$. L'émission de feuilles primaires correspond à une phase démographique linéaire alors que celle des feuilles secondaires est assurément exponentielle (fig. 5).

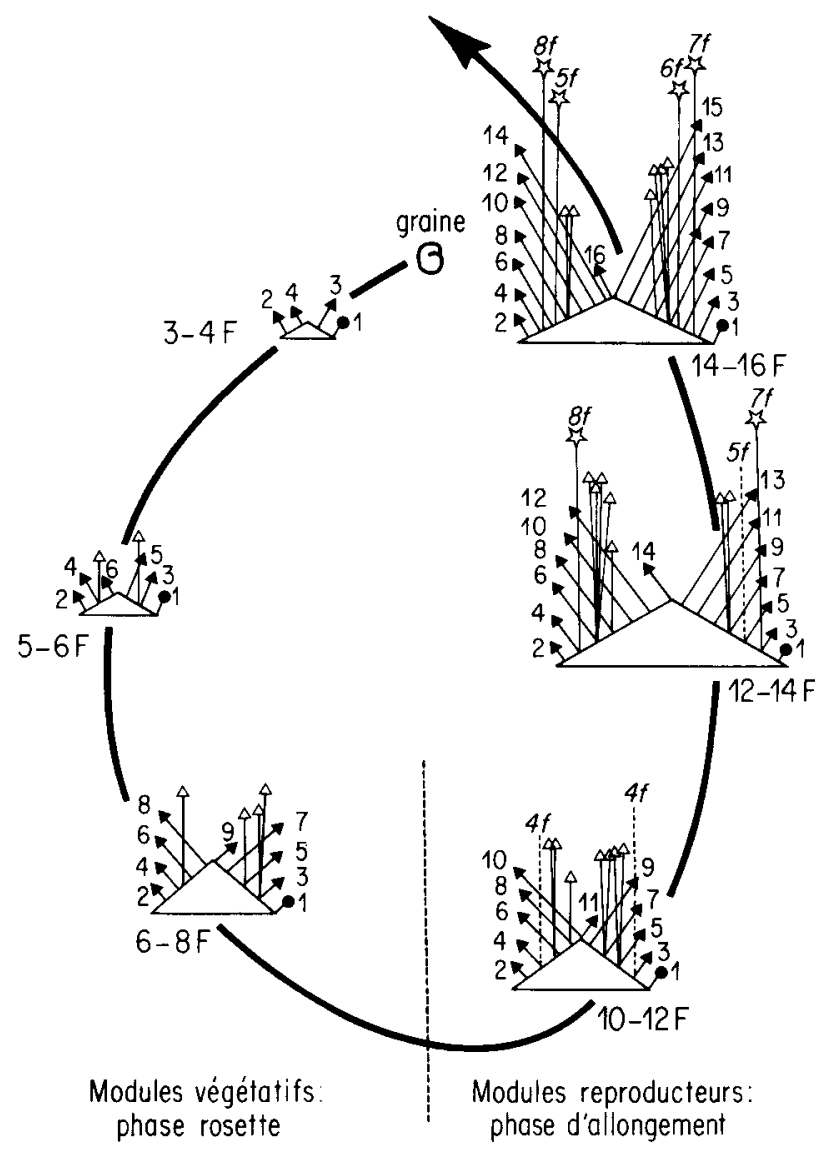

Figure 4

Schéma d'évolution de la $1^{\text {re }}$ cohorte de modules chez Trifolium pratense $L$.

Change with time of the first cohort of modules.

- Feuille primaire $F_{1}$ non trifoliée.

Non-trifoliate primary leaf.

- Feuilles primaires trifoliées avec ordre d'émergence. Trifoliate primary leaves with order of emergence.

3-4F. Nombre total de feuilles primaires de la plante.

Total number of primary leaves of the plant.

If $\triangle$ feuilles secondaires $(f)$ issues d'un bourgeon axillaire, ramean i) en allongement avec nombre de feuilles secondaires (5f).

i) Secondary leaves (f) from an axillary bud, branch with number i) of secondary leaves $(5 f)$.

$7 f$ ? rameau fleuri avec nombre de feuilles secondaires (7f). \} flowered branch with number of secondary leaves (7f).

Durant cette période d'émission de feuilles la plante présente une structure se situant dans un plan quasi vertical. Plus tard, mais avant la phase d'allongement, les modules secondaires dévient dans un plan perpendiculaire à celui des modules primaires pour s'insérer dans un espace libre ; la plante prend alors la véritable forme d'une rosette (fig. 6).

La réitération modulaire entraîne une expansion du nombre de modules. Partant d'une plantule qui peut être considérée dès l'acquisition de la feuille $F_{1}$ comme " le module initial », nous obtenons au moment de la récolte un nombre élevé mais variable de modules selon les traitements expérimentaux. Les différences s'estompent au cours du temps, la mortalité étant plus élevée que la natalité modulaire à forte densité ; le nombre de modules par parcelle tend vers un équilibre 
ne dépendant uniquement que de la proportion initiale des espèces associées (fig. 7), c'est-à-dire du nombre de lignes de chaque partenaire dans l'association. La densité linéaire tend vers une densité unique de 130 modules/m à l'issue d'une évolution qui, dans le cas de la $2^{\mathrm{e}}$ expérience, comprend 5 périodes de repousse sur environ $450 \mathrm{j}$ de culture. Cette tendance dans l'évolution de la densité modulaire a été notée par

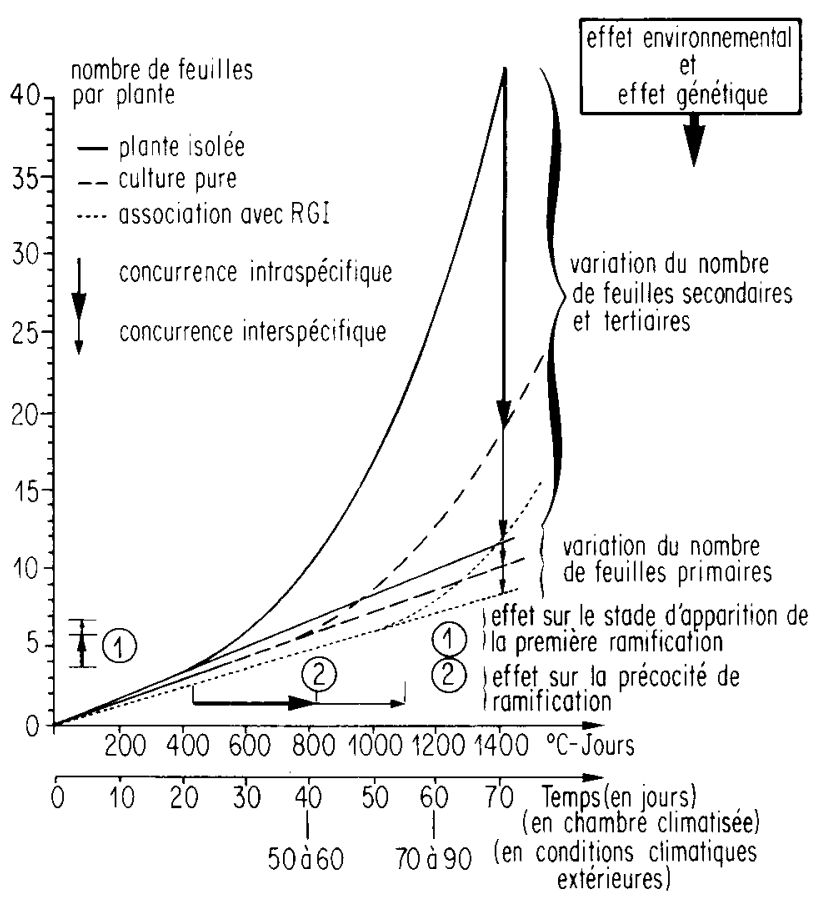

Figure 5

Représentation schématique de la croissance de la population de feuilles d'un pied de Trifolium pratense $L$. et de l'effet de la concurrence de plantes voisines lors de la $1^{\text {re }}$ pousse ( $I^{\text {re }}$ cohorte de feuilles). Graph of the growth of the leaf population from a shoot of Trifolium pratense and effect of competition from adjacent plants during the Ist growth (Ist cohort of leaves).

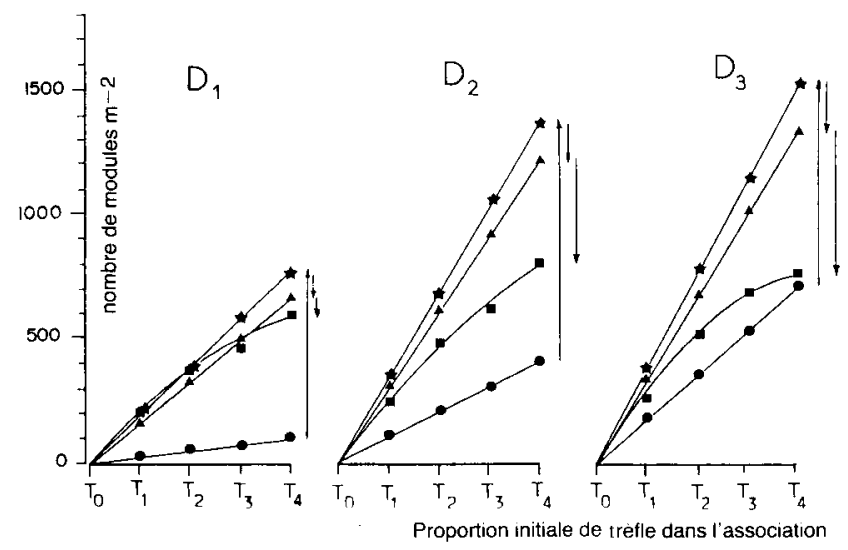

Figure 7

Evolution sur 2 années de culture au champ de la densité moyenne de modules de trèfle violet selon la densité $\left(D_{1}, D_{2}, D_{3}\right)$ et la proportion des partenaires au semis. $C v$. «Rotra " en culture pure $\left(T_{4}\right)$ et en association $\left(T_{1}, T_{2}, T_{3}\right)$ avec le ray-grass d'Italie $c v$. «Adret ".

Change over two years, for a field crop, of the mean density of modules according to the density and to the proportion of partners at sowing. $C v$. 'Rotra' in pure stand $\left(T_{4}\right)$ and in association $\left(T_{1}, T_{2}\right.$, $T_{3}$ ) with Italian rye-grass (cv. 'Adret').

$D_{1}, D_{2}, D_{3}=100,400,700$ plantes $/ \mathrm{m}^{2}$

$T_{0}, T_{1}, T_{2}, T_{3}, T_{4}=0,25,50,75,100 \mathrm{p} .100$ de trèfle violet (Red clover).

- Le 24 avril 1978: levée de trèfle (une feuille primaire), une plantule $=$ un module initial.

Establishment of the clover (one primary leaf), one seedling = one initial module.

$\star$ début avril $1979=$ départ en végétation. starting of growth.

$\Delta$ le 10 juin $1979=2$ jours avant la récolte $C_{4}$. 2 days before cutting.

le 16 juillet $1979=2$ jours avant la récolte $C_{5}$. 2 days before cutting. amplitude et sens de la variation de la densité modulaire.

range and direction of variation of module density.

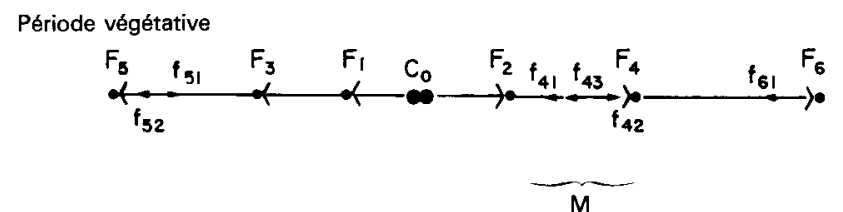

\section{Figure 6}

Organigramme d'une plante de Trifolium pratense $L$. en $I^{\text {re }}$ cohorte de modules.

La position de chaque feuille est représentée par ses stipules $(\alpha)$.

Graph of an individual for the 1st cohort of modules.

The situation of each leaf is indicated by its stipules.

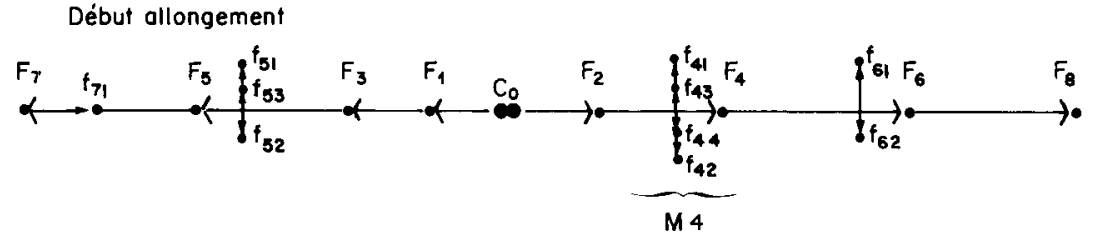

$\alpha$

$F_{S}$ feuille primaire Co $=$ cotylédons

$\kappa$ primary leaf

$f_{43}$ feuille secondaire secondary leaf

$M_{4}$ Module primaire primary module 
certains démographes travaillant sur des cohortes de talles de Phleum pratense L. (LANGER, 1956 ; GARWOOD, 1969, in WHITE, 1980).

Les facteurs de variation étudiés dans nos expériences (MAITRE \& JACQUARD, 1980) vont dans le même sens qu'une gêne de la ramification. On note un rythme d'émergence légèrement plus lent pour les feuilles primaires lorsque l'on passe de la culture pure à l'association avec une graminée tout au moins avec du ray-grass d'Italie (fig. 5) ; il en est de même lorsque la densité augmente en culture pure. Cet écart est de l'ordre d'une feuille primaire, ce qui correspond à 5-6 $\mathrm{j}$ de retard dans l'organisation de la plante à la récolte. Par contre, les effets sur l'émergence - et donc le nombre de feuilles secondaires - sont beaucoup plus importants (fig. 5 et 8 , tabl. 2). La concurrence,
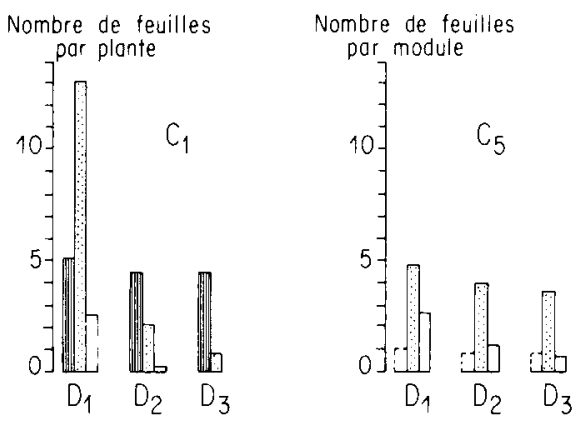

Figure 8

Nombre moyen de feuilles par individu (plante ou module) de trèfle violet et par catégorie foliaire (primaires, secondaires, tertiaires) lors des $1^{r e}\left(C_{1}\right)$ et $5^{e}$ coupes $\left(C_{5}\right)$ selon la densité initiale de semis $\left(D_{1}\right.$, $\left.D_{2}, D_{3}\right)$. Moyenne de 120 plantes ou modules par densité, blocs et proportions de partenaires au semis (confondus).

Mean number of leaves per individual (plant or module) of red clover, and per leaf category (primaries, secondaries, tertiaries) for the first $\left(C_{1}\right)$ and fifth $\left(C_{5}\right)$ cuttings, according to the initial sowing density $\left(D_{1}, D_{2}, D_{3}\right)$. Mean of 120 plants or modules per density, blocks and ratios of partners at sowing (confounded).

$D_{1}, D_{2}, D_{3}=100,400,700$ plantes $/ \mathrm{m}^{2}$.

\section{feuilles primaires \\ feuilles secondaires \\ feuilles tertiaires}

(primary leaves)

(secondary leaves)

(tertiary leaves)

Dès la $2^{e}$ cohorte, toute feuille parentale issue de la cohorte précédente et générant une lignée de modules est qualifiée de primaire; elle est donc unique par module étudié et en général morte à la récolte (-

From the second cohort, any parental leaf originating in the preceding cohort and generating a strain of modules is considered as a primary leaf ; it is unique per module studied and commonly, dead at the harvest (

\section{TABLEAU 2}

Nombre moyen de feuilles secondaires par module en fonction de la densité (plantes $/ \mathrm{m}^{2}$ ) et de l'ordre du module parental en $1^{r e}$ cohorte. Mean number of secondary leaves per module in relation to density $\left(\mathrm{pl} / \mathrm{m}^{2}\right)$ and order of the parental module in the first cohort.

\begin{tabular}{lccc}
\hline \hline \multicolumn{1}{c}{ Densité } & & & \\
Module & 100 & 400 & 700 \\
\hline $\mathrm{M}_{3}$ & 2,80 & 0,13 & 0,03 \\
$\mathrm{M}_{4}$ & 3,96 & 0,75 & 0,15 \\
$\mathrm{M}_{5}$ & 3,69 & 0,73 & 0,41 \\
$\mathrm{M}_{6}$ & 1,80 & 0,20 & 0,06 \\
$\mathrm{M}_{7}$ & 0,60 & 0,08 & 0,03 \\
\hline \hline
\end{tabular}

qu'elle soit d'origine intraspécifique ou interspécifique, entraîne un retard stadial et spatial dans l'émission de modules secondaires. Cela se traduit à la récolte par une baisse du nombre total de feuilles, soit 5 à 10 feuilles en moyenne par individu, ainsi que par une structure différente (tabl. 2).

Comme toute population naturelle, la population foliaire d'un pied de trèfle tend vers une valeur limite supérieure; le taux de croissance réel diminue puis devient nul lorsque l'effectif foliaire a atteint la capacité biotique du milieu pour la population considérée. En l'absence de perturbation, de contrainte ou de concurrence, cette limite est imposée par la floraison.

L'équation logistique de Verhulst (BAZZAZ \& HARPER, 1977 ; HARPER, 1977) appliquée à la population de feuilles du trèfle violet s'écrit :

$$
\frac{\mathrm{dN}}{\mathrm{dt}}=\mathrm{r}_{\mathrm{m}} \mathrm{N}\left(\frac{\mathrm{K}-\mathrm{N}}{\mathrm{K}}\right)
$$

$r_{\mathrm{m}}=$ taux intrinsèque de croissance de la population de feuilles que HARPER (1977) définit également comme " le taux intrinsèque de naissance foliaire ".

Mathématiquement $r_{m}$ représente une vitesse instantanée de développement. On peut considérer ce paramètre comme une mesure du taux relatif potentiel d'accroissement de la population (BARBAULT, 1981).

$\mathrm{K}=$ nombre total de feuilles émises par la plante ; ici l'émission de feuilles est interrompue par la récolte. $\mathrm{K}$ représente donc en fait le nombre de feuilles émises jusqu'à la récolte.

$\mathrm{N}=$ nombre de feuilles émises jusqu'à la date $\mathrm{t}$.

La forme intégrée de l'équation logistique peut s'écrire :

$$
\mathrm{N}_{\mathrm{t}}=\frac{\mathrm{K}}{1-\mathrm{e}^{\mathrm{a}-\mathrm{r}_{\mathrm{m}} t}}
$$

et permet de calculer la valeur du paramètre $r_{m}$ par :

$$
\log _{\mathrm{e}}\left(\frac{\mathrm{K}-\mathrm{N}}{\mathrm{N}}\right)=\mathrm{a}-\mathbf{r}_{\mathrm{m}} \mathrm{t} \quad \text { avec } \mathrm{a}=\text { constante } \text {. }
$$

La figure 9 montre très nettement l'effet de la concurrence sur la valeur du paramètre $r_{m}$ dans la phase correspondant à l'émission de feuilles secondaires dans le cas de structures génotypiques différentes (1 ${ }^{\text {re }}$ expérience). On y voit également que l'incertitude sur $r_{m}$ est probablement faible. Cette figure indique également que, si la structure génotypique ne modifie pas la valeur du paramètre, elle conditionne la précocité de ramification. En effet, 3 constatations s'imposent :

- les taux intrinsèques de croissance sont identiques en amont de la rupture de pente pour les 3 familles,

- en aval de la rupture de pente, ces taux sont également identiques pour les familles en culture pure, ils sont identiques aussi en association mais, dans ce cas, leurs valeurs sont plus faibles qu'en culture pure,

- si l'on cherche à quoi peut correspondre la rupture de pente, on s'aperçoit qu'elle est en rapport avec le stade feuillu de la plante, lequel correspond à la $1^{\text {re }}$ ramification.

La précocité de ramification détermine en partie la résistance du trèfle, au stade jeune, et éventuellement 


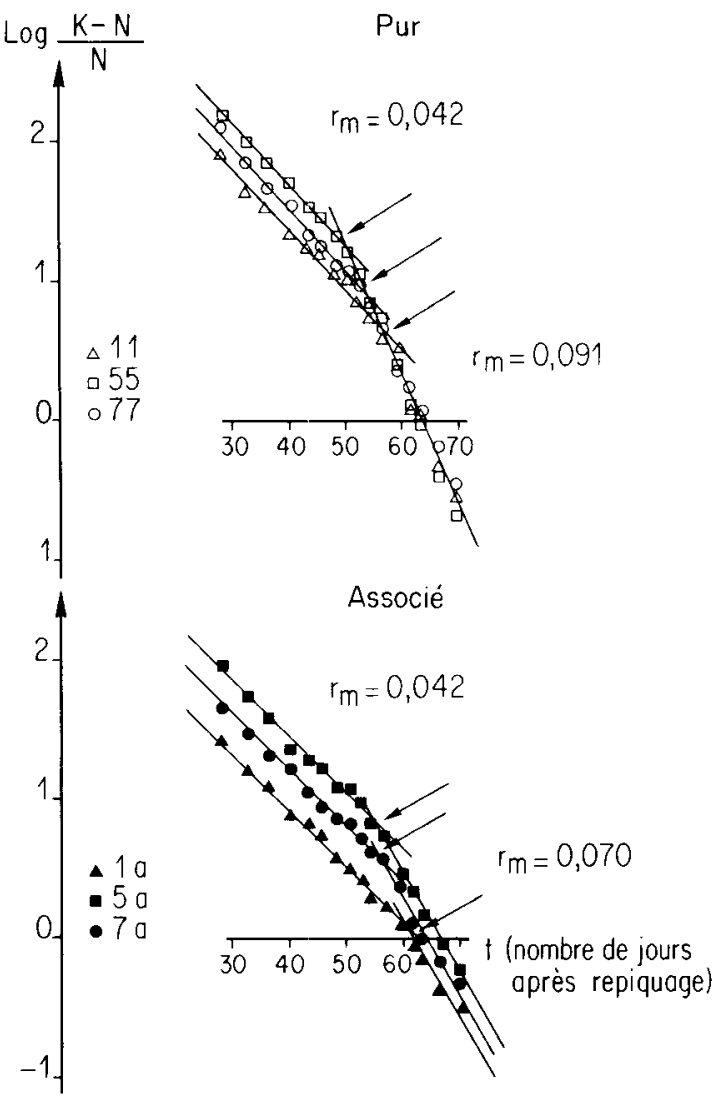

Figure 9

Relation entre $\log _{e}\left(\frac{K-N}{N}\right)$ et le temps pour la population de feuilles d'un pied moyen de trèfle violet (familles $1,5,7)$ cultivé en pur ou en association avec le ray-grass d'Italie cv. «Tiara 》 (a). Cultures en pots et conditions extérieures. Moyenne de 12 plantes par famille et par culture.

$11,55,77=$ Cultures pures de chaque famille.

Ia, $5 a, 7 a=$ En association avec cv. Tiara $(a)$.

La flèche indique pour chaque famille la rupture de pente correspondant au début d'émission des feuilles secondaires.

$r_{m}=$ Taux intrinsèque de croissance de la population.

$K=$ Nombre total de feuilles émises jusqu'à la récolte.

$N=$ Nombre total de feuilles émises jusqu'à la date $t$.

Relationship between $\log _{e}\left(\frac{K-N}{N}\right)$ and time for the population of leaves of an average individual of red clover, grown in pure stand or in association with Italian rye-grass, cv. 'Tiara' (a). Growth in pots and outdoors. Mean of 12 plants/family/condition of growth.

$11,55,77=$ Pure stands.

la, $5 a, 7 a=$ Associations.

The arrow shows for each family the break in slope corresponding to the start of secondary leaf emergence.

$r_{m}=$ Intrinsic growth rate for the population of leaves.

$K=$ Total number of leaves emerged until cutting.

$N=$ Total number of leaves emerged until time $t$.

au-delà, à l'agressivité de la graminée (MAITRE, 1977, 1981).

\section{L'état reproducteur}

Lorsque la rosette possède 8 à 10 feuilles primaires, l'allongement des entre-nœuds commence, la phase reproductrice est alors amorcée et, à 10 à 12 feuilles primaires, les rameaux sont visibles. En ce qui concerne l'organisation de la plante à la floraison, nos

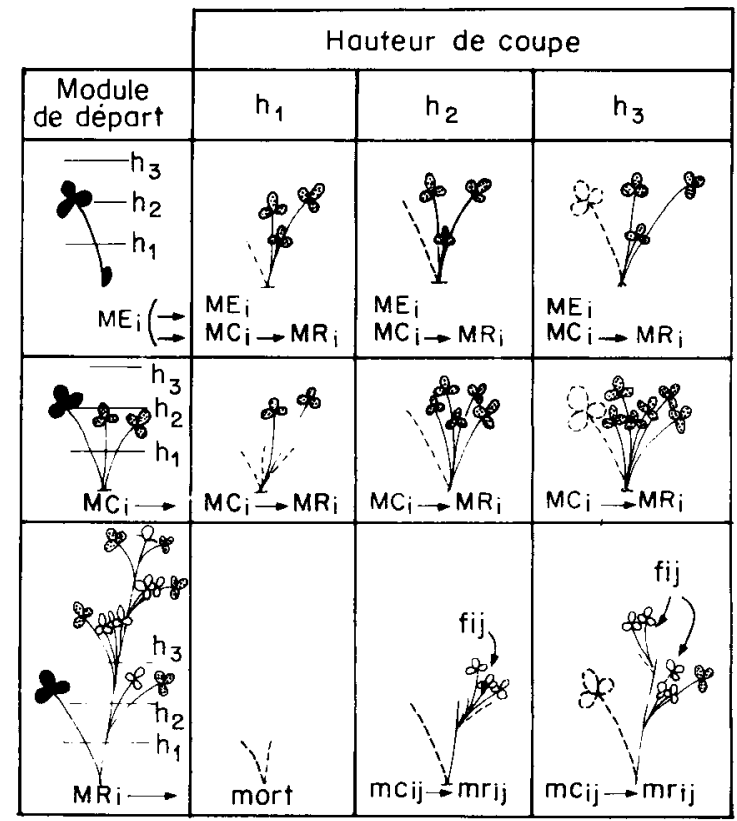

Figure 10

Représentation schématique des évolutions possibles d'un module parental (primaire par exemple) selon son organisation de départ et la hauteur de coupe. Il n'a pas été tenu compte des éventuelles corrélations entre modules au sein de la colonie, de ce fait, la mort du module parental est toujours possible.

Diagram of changes with time possibly occurring for a parental module (i.e. a primary one), according to its initial organization and to cutting height. Possible correlations between modules within the colony are not taken into account, so the death of the parental module is always possible.

Module primaire :

Module secondaire :

(primary module)

$M_{E i}=$ module élémentaire (elementary module)

$M_{C i}=$ module composé (secondary module) $M_{c i j}=$ module composé (composite module)

$M_{r i j}=$ module reproducteur

$M_{R i}=$ module reproducteur (reproductive module)

$M_{C i} \longrightarrow M_{R i}=$ évolution après la coupe (change after cutting)

$\because$ organe mort

$\because$ (dead organ

$\boldsymbol{X}_{F_{i}}$

$\mathscr{R} f_{i j}$

$\& f_{i j k}$

$h_{1}, h_{2}, h_{3}=$ hauteur de coupe $(2 \dot{a} 8 \mathrm{~cm}$ en moyenne) (cutting height).

observations sont en accord avec celles de VESCOVI (1977), seul auteur, à notre connaissance, à s'être préoccupé de cet aspect. En effet, pour la $1^{\text {re }}$ cohorte de modules, nous remarquons que la mise à fleurs intervient après l'émission d'un même nombre de feuilles secondaires pour un rameau donné. Cette caractéristique est fonction du rang du module primaire ayant initialisé ce rameau (fig. 4). Il faut par exemple 67 feuilles secondaires pour que le module primaire $\mathrm{M}_{5}$ donne un rameau fleuri, mais il en faut 5-6 pour le module $\mathrm{M}_{6}$. 
Contrairement à ce que l'on observe chez les graminées où la précocité de floraison de la touffe est centrifuge, chez le trèfle violet elle est centripète (fig. 4). Il n'y a donc pas de tige maitresse comme semble l'indiquer VESCOVI (1977), mais un rameau qui fleurit en premier, en général celui du module $\mathrm{M}_{4}$.

La suppression simultanée des 2 premières feuilles primaires $\left(F_{1}\right.$ et $\left.F_{2}\right)$ de la plantule stimule la floraison si l'exposition préalable aux conditions d'éclairement (jours longs) nécessaires à l'induction florale a été respectée (JONES \& STODDART, 1972). D'après ces auteurs, il faudrait 9 à $11 \mathrm{j}$ d'éclairement à $16 \mathrm{~h}$ lorsque la plantule a 2 feuilles primaires pour obtenir la floraison. Le contrôle génétique de cette réponse serait intéressant à connaître.

Le rôle physiologique particulier des 2 premières feuilles primaires (JONES \& STODDART, 1973) pourrait expliquer le fait que leurs bourgeons axillaires ne développent jamais de feuilles secondaires et qu'il y ait très souvent concordance entre le démarrage de l'émission des feuilles secondaires et la mort de la $\mathrm{F}_{1}$ (MAITRE \& JACQUARD, 1980).

\section{Effet d'une coupe sur l'organisation morphologi- que ultérieure}

La structure de la touffe du trèfle violet après une coupe est, pour une grande part, le résultat de l'interaction entre la proportion respective de chaque type de module présent avant la coupe $(\S 1 b)$ et la hauteur de la coupe (fig. 10).

Tout module élémentaire demeurant en place dans la colonie peut émettre par la suite un nombre de modu- les fils en rapport avec les nouvelles corrélations apparues entre les modules restants. Seuls les modules reproducteurs sectionnés trop bas ne donneront pas de descendance, sauf dans le cas où le port prostré des rameaux leur permet d'échapper partiellement à la coupe. Assez rarement, surtout dans le cas de port prostré, on constate une émission de racines adventives au niveau des premiers nœuds contribuant ainsi à l'extension de la colonie.

\section{CONCLUSION}

Un pied de trèfle violet peut être décrit comme une population d'individus de base appelés « modules». Chaque module constitué d'une feuille et de son bourgeon axillaire a une vie propre conditionnée par son ordre d'apparition dans la colonie (naissance, croissance, multiplication, mort).

La conception modulaire confère au trèfle violet une analogie démographique avec le tallage des graminées et permet de mieux décrire sa plasticité. Elle suggère des voies d'approche différentes dans l'étude des phénomènes de croissance à l'échelle de la parcelle, considérée comme une population de modules.

Une bonne connaissance de la mise en place de l'organisation morphologique du trèfle et de la réponse de cette organisation aux conditions d'association était un préalable à l'analyse des relations entre démographie des organes et climat qui fera l'objet du $2^{\mathrm{e}}$ article de cette série.

Reçu le 20 avril 1984 Accepté le 25 octobre 1984

\section{RÉFÉRENCES BIBLIOGRAPHIQUES}

Barbault R., 1981. Ecologie des populations et des peuplements. Masson, Paris, 200 p.

Bazzaz F. A., Harper J. L., 1977. Demographic analysis of the growth of Linum usitatissimum. New Phytol., 78, 198-208.

Blackmann V. H., 1919. The compound interest law of plant growth. Ann. Bot., 33, 353.

Evans G. C., 1972. The Quantitative Analysis of Plant Growth. Blackwell Sci. Publ. Oxford, 734 p.

Garwood E. A., 1969. Seasonal tiller populations of grass and grass/clover swards with and without irrigation. J. Br. Grassl. Soc., 24, 333-344 (cité par WhITE, 1980).

Gillet M., 1970. Sur quelques aspects de la croissance et du développement de la plante entière de graminée en conditions naturelles: Festuca pratensis Huds. Thèse d'Etat, Poitiers, 190 p. et 39 annexes.

Gillet M., 1980. Les graminées fourragères : description, fonctionnement, applications à la culture de l'herbe. Gauthier-Villars, Paris, $306 \mathrm{p}$.

Gregory F. G., 1917. Physiological conditions in cucumber houses. Cheshunt (England), Exp. and Res. Stp., 3rd Annu. Rep., 19.

Harper J. L., 1977. Population Biology of Plants. Acad. Press., London, $892 \mathrm{p}$.

Hruskova H., 1973. (Morphological structure of the tillering zone in Medicago sativa L.. I. Tillering zone from sowing to the first cut), (en tchèque). Sb. Ved. Pr., 3, 9-17.

Hunt R., 1978a. Plant growth analysis. Studies in Biology, Edward Arnold, London, $67 \mathrm{p}$.

Hunt R., 1978b. Demography versus plant growth analysis. New Phytol., 80, 269-272.

Jones T. W. A., Stoddart J. L., 1972. Effects of leaf removal on flowering in red clover. Rep. Welsh Plant Breed. Stn., 79-80.
Jones T. W. A., Stoddart J. L., 1973. Physiology of flowering in red clover. Rep. Welsh Plant Breed. Stn., 52-53.

Langer R. H. M., 1956. Growth and nutrition of timothy (Phleum pratense). The life history of individual tillers. Ann. appl. Biol., 44, 166-187 (cité par WhITE, 1980).

Maitre J.-P., 1977. Aptitude à la vie en association chez le trèfle violet. Ann. Amélior. Plantes, 17 (3), 369-387.

Maitre J.-P., 1981. Dynamique des populations d'organes et de la production de matière sèche chez Trifolium pratense $L$. Rôle de la concurrence. Thèse Doct. Ing., Fac. Sci., Montpellier, 112 p. et 108 annexes.

Maitre J.-P., Jacquard P., 1980. Etude préalable à la modélisation de la production primaire du trèfle violet associé au ray-grass d'Italie : réponse de l'organisation morphologique aux conditions d'association. C.R. de fin de recherches D.G.R.S.T., Ronéo, 20 pages et 22 annexes.

Mallet P., 1979. Liaison statistique entre le développement et la croissance chez les plantes : application d'un modèle simplifié à l'analyse de croissance. Ann. agron., 30 (5), 415-430.

Radford J., 1967. Growth analysis formulae. Their use and abuse. Crop Sci., 7, 171.

Vescovi P., 1977. Influence de différentes températures sur la mise à fleur du Trifolium pratense L. C.R. Acad. Sci. Paris, 284 p., série $\mathrm{D}, 1075-1078$.

White J., 1980. Demographic factors in populations of plants, 21-48. In O. T. Solbrig « Demography and evolution in plant populations ». Botanical Monographs (15), Blackwell Scientific Publications.

Wit C. T. (de), 1960. On competition. Versl. Landbowck. Onderz., $66(8), 1-82$. 